\title{
The importance of the Proceedings in Thoracic Endoscopy of the AIPO Study Group of Interventional Pulmonology
}

\author{
M. Patelli1, B. Balbi2
}

Monaldi Arch Chest Dis 2011; 75: 1, 6-7.

Keywords: Interventional pulmonology, Bronchoscopy, Thoracoscopy, Guidelines, Cancer, Diffuse parenchymal lung disorders.

1 U.O. Endoscopia Toracica e Pneumologia, Dipartimento di Scienze Oncologiche, Ospedali Maggiore e Bellaria, Bologna,

2 Divisione di Pneumologia Riabilitativa, Fondazione Salvatore Maugeri, I.R.C.C.S., Veruno (Novara), Italy.

Correspondence: Dr. Marco Patelli, U.O. Endoscopia Toracica e Pneumologia, Dipartimento di Scienze Oncologiche, Ospedale Maggiore, Largo Nigrisoli 2, 40100 Bologna, Italy; e-mail: marco.patelli@ausl.bologna.it

Interventional pulmonology procedures have become a mainstay in the scientific background and in clinical practice of respiratory physicians. They allow for the diagnosis of a number of different conditions involving airways, parenchyma and mediastinum, as well as to relieve symptoms related to malignant and benign obstructions of the central airways.

During the last decade, several efforts were made by scientific societies composed of interventional pulmonologists to accurately define both diagnostic and therapeutic invasive procedures in the setting of accreditation and certification processes.

In 2006 the Italian Society of Pulmonologists (AIPO: Associazione Italiana Pneumologi Ospedalieri) endorsed a Consensus Conference aimed at discussing the operative protocols concerning all the areas of interventional pulmonology. The conference, that took place in Bologna on 2-3 March 2006, was attended by approximately 150 participants and a limited number of speakers. Each speaker was required to revise the literature and to propose a few recommendations on a specific topic to be discussed with the other speakers and the attendees. Recommendations were graded, when applicable, using an adapted scale used by the US Preventive Services Task Force (USPSTF) in the BTS guidelines on diagnostic flexible bronchoscopy (Table 1) [1]. In the text of each article, grades of recommendations are reported if appropriated and levels of evidence are presented also in the text of the majority of the articles.

The articles offered in the current monographic issue of Monaldi Archives for Chest Diseases (MACD) present and discuss briefly the recommendations which were produced, by consensus, in the aforementioned conference, with some modifications if appropriated.

What is the relevance of such proceedings to the reader of MACD? In the last ten years only the BTS issued their official guidelines, while other major Scientific Societies did not: ACCP [2] and
Table 1. - Levels of evidence and grading of recommendations

Level Type of evidence

Ia Evidence obtained from meta-analysis of randomised controlled trials

Ib Evidence obtained from at least one randomised controlled trial

IIa Evidence obtained from at least one well designed controlled study without randomisation

IIb Evidence obtained from at least one other type of well designed quasi-experimental study

III Evidence obtained from well designed non-experimental descriptive studies such as comparative studies, correlation studies, and case controlled studies

IV Evidence obtained from expert committee reports of opinions and/or clinical experiences of respected authorities

Grade Type of recommendations

A (levels Ia, Ib) Requires at least one randomised controlled trial as part of a body of literature of overall good quality and consistency addressing the specific recommendation

B (levels IIa, IIb, III) Requires availability of well conducted clinical studies but no randomised clinical trials on the topic of recommendation

C (level IV) Requires evidence from expert committee reports or opinions and/or clinical experience of respected authorities. Indicates absence of directly applicable studies of good quality

Modified from [1]. 
ERS/ATS [3] Task Force of European and north American experts published position papers to define the field of Interventional Pulmonology as well as to describe the minimal requirements in methodology and logistics, the indications, contraindications, outcomes and training requirements for each interventional procedure. These documents however can not be correctly definied as Guidelines. Our aim is therefore to supply the readers with an example of Guidelines in an important field of Pulmonology made by our National Society, in the hope that this document will foster new initiatives at an European and/or International level.

\section{References}

1. British Thoracic Society Bronchoscopy Guidelines Committee, a Subcommittee of Standards of Care Committee of British Thoracic Society. British Thoracic Society guidelines on diagnostic flexible bronchoscopy. Thorax 2001; 56 Suppl 1: i1-21.

2. Ernst A, Silvestri GA, Johnstone D; American College of Chest Physicians. Interventional Pulmonary Procedures: Guidelines from the American College of Chest Physicians. Chest 2003; 123: 1693-1717.

3. Bolliger CT, Mathur PN, Beamis JF, et al. ERS/ATS Statement on Interventional Pulmonology. Eur Resp $J$ 2002; 19: 356-373.

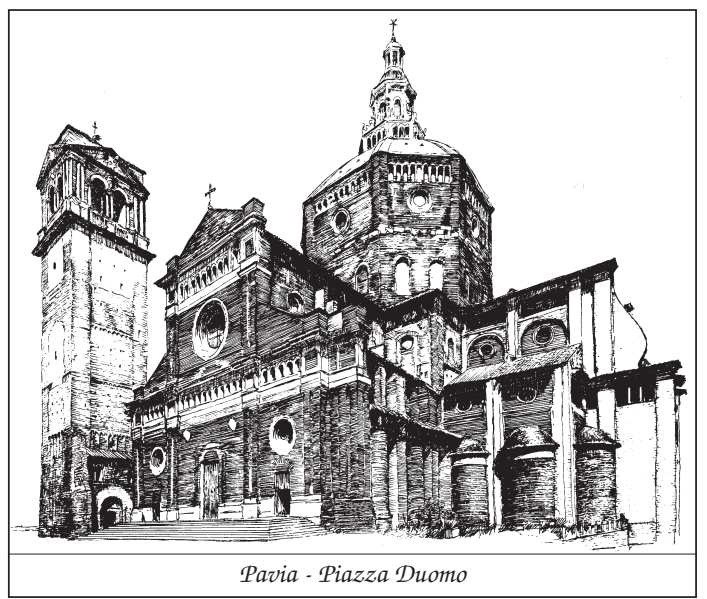

